\title{
Induction of Canonical Wnt Signaling by Synovial Overexpression of Selected Wnts Leads to Protease Activity and Early Osteoarthritis-Like Cartilage Damage
}

Martijn H. van den Bosch, Arjen B. Blom, Annet W. Sloetjes, Marije I. Koenders, Fons A. van de Loo, Wim B. van den Berg, Peter L. van Lent, and Peter M. van der Kraan

Accepted for publication March 24, 2015.

Address correspondence to Arjen B. Blom, Ph.D., Radboud University Medical Center, Geert Grooteplein 28, 6525 GA Nijmegen, the Netherlands. E-mail: arjen.blom@radboudumc.nl.

\begin{abstract}
Proteins from the Wnt signaling pathway are very important for joint development. Curiously, osteoarthritis $(\mathrm{OA})$ is thought to be a recapitulation of developmental processes. Various members of the Wnt signaling pathway are overexpressed in the synovium during experimental OA. Here, we investigated the potency of specific Wnt proteins, when expressed in the synovium, to induce OA pathology. We overexpressed Wnt5a, Wnt8a, Wnt16, and WISP1 in the synovium using adenoviral vectors. We determined whether overexpression resulted in OA pathology by histology, and we measured whether Wnt signaling led to increased protease activity in the joint. Synovial overexpression of Wnt8a and Wnt16 led to canonical Wnt signaling in the cartilage, whereas overexpression of Wnt5a did not. Canonical Wnt signaling increased protease activity and induced cartilage damage shortly after overexpression. Specific blocking of the canonical Wnt signaling pathway with Dickkopf-1 reduced the Wnt-signaling-induced cartilage damage. By contrast, the noncanonical signaling Wnt5a did not cause cartilage lesions. Overexpression of WISP1, a downstream protein of canonical Wnt signaling, resulted in increased cartilage damage. In conclusion, our data show that canonical Wnts and WISP1, which we found overexpressed in the synovium during experimental $\mathrm{OA}$, may conduce to $0 \mathrm{~A}$ pathology. (Am J Pathol 2015, 185: 1970-1980; http://dx.doi.org/10.1016/j.ajpath.2015.03.013)
\end{abstract}

The Wnt signaling pathway plays a central role in a plethora of processes, including embryonic development, axis patterning, cell proliferation, and cell migration. The development of the articular joints, including cartilage, bone, and joints cavities, is highly dependent on Wnt signaling. ${ }^{1-3}$ Osteoarthritis (OA) is a degenerative joint disease with cartilage damage, synovial inflammation, fibrosis, and osteophyte formation. OA probably is in part the result of a recapitulation of developmental processes in the joint, making Wnt signaling an alluring pathway to study.

In the past, we have found highly increased expression of several Wnts specifically in the synovium of various murine experimental OA models, as well as an increase in WNT1inducible signaling pathway protein 1 (WISP1), a downstream protein of canonical Wnt signaling, in both the synovium and the cartilage. In addition, increased expression of WISP1 was found in human OA cartilage.

Wnt proteins are a family of lipid-modified glycoproteins that can bind to Frizzled (Fzd) receptors. Wnt proteins can signal via noncanonical and canonical signaling pathways. Although the paradigm is shifting, Wnt5a is historically considered to be a noncanonical protein, whereas Wnt8a is considered a canonical Wnt protein. In addition to $\mathrm{Wnt}-\mathrm{Fzd}$ interaction, binding to the coreceptor complex of low-density lipoprotein receptor-related protein (LRP) $5 / 6$ is essential for $\beta$-catenin-dependent canonical signaling.

Supported by Dutch Arthritis Foundation grant 08-1-309 (A.B.B.). Disclosures: None declared. 

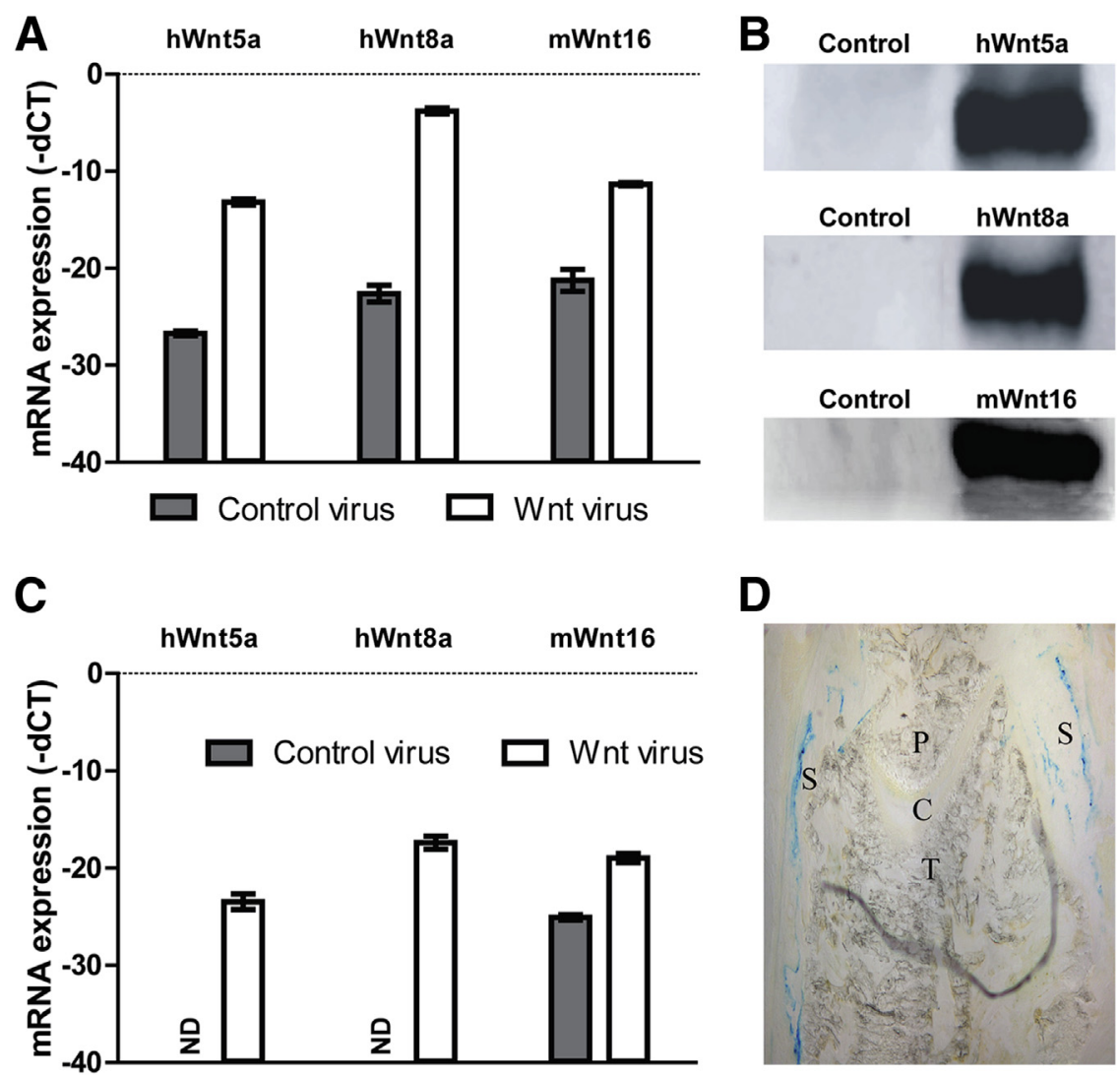

Figure 1 Adenoviral targeting resulted in increased expression of Wnt5a, Wnt8a, and Wnt16. A: Quantitative real-time PCR showed that targeting of primary murine synovial fibroblasts with adenoviral vectors containing Wnt5a, Wnt8a, and Wnt16 results in increased expression of these Wnts at the RNA level, 24 hours after overexpression. B: Western blot showed increased protein expression. C: Intra-articular injection of adenoviral vectors containing Wnt5a, Wnt8a, and Wnt16 into murine knee joints resulted in increased expression of these members of the Wnt signaling pathway in the synovium 3 days after overexpression. D: In vivo overexpression with the adenoviral vectors resulted in increased expression specifically in the synovium, but not in the cartilage. Representative Western blots and a representative image of an X-Gal staining are shown. Values are presented as $-\mathrm{dC}_{\mathrm{T}}$ means $\pm \mathrm{SEM}$, indicated by the error bars. $C$, cartilage; ND, not detectable; $P$, patella; $S$, synovium; $T$, tibia.
Normally, $\beta$-catenin is continuously ubiquitinated by a protein complex and subsequently broken down, a process that is inhibited by binding of a Wnt protein to a Fzd receptor and LRP5/6 leading to translocation of $\beta$-catenin to the nucleus where it converts TCF/LEF to an activator of transcription.

Most of the data concerning the role of Wnt signaling in $\mathrm{OA}$ is related to the canonical pathway. Polymorphisms in the genes encoding various members of the Wnt signaling pathway have been linked to OA. ${ }^{8-11}$ In addition, various studies with conditional activation or inhibition of Wnt signaling in the cartilage show effects on disease activity. ${ }^{12-14}$

Active Wnt signaling has been shown to result in increased expression of matrix metalloproteinases (MMPs) in various diseases, including many types of tumors. ${ }^{15-17}$ Among the MMPs that are increased by Wnt signaling are MMP2, MMP3, MMP9, and MT1-MMP, and in addition, Wnt signaling increases the expression of the aggrecanases A disintegrin and metalloproteinase with thrombospondin motifs (ADAMTS) 4 and $5 .{ }^{13,18-21}$ Breakdown of the matrix surrounding the chondrocytes by protease activity is one of the main mechanisms underlying the damage that is found in OA. During arthritic diseases, both MMPs and aggrecanases cleave aggrecan, one of the most abundant proteins in the cartilage matrix. This leads to the formation of the neoepitopes VDIPEN and NITEGE, respectively. ${ }^{22-24}$
Here, we investigated the capability of members of the Wnt signaling pathway to cause cartilage damage in vivo in naive mice. We intra-articularly injected adenoviral vectors containing several selected members of the Wnt signaling pathway into murine knee joints to overexpress these proteins, after which, the knee joints were examined for OA-like pathology by histological analysis and immunohistochemical staining to reveal the presence of protease activity.

\section{Materials and Methods}

\section{Animals}

Male C57BL/6 mice (12 weeks old) were purchased from Janvier (Le Genest Saint Isle, France). All mice were housed in filtertop cages with woodchip bedding. Mice were fed a standard diet, with food and tap water ad libitum. All animal studies were according to the Dutch law and approved by the local animal experimentation committee.

\section{Cell Lines and Culture}

Primary synovial fibroblasts were isolated from the synovial tissue of C57B1/6 mice by spontaneous outgrowth for 2 weeks and were cultured in Dulbecco's modified Eagle's medium (Invitrogen, Carlsbad, CA) supplemented with $10 \%$ fetal calf serum (Hyclone, Logan, UT), sodium pyruvate, and $48 \mu \mathrm{g} / \mathrm{mL}$ gentamicin at $37^{\circ} \mathrm{C}$. 
A
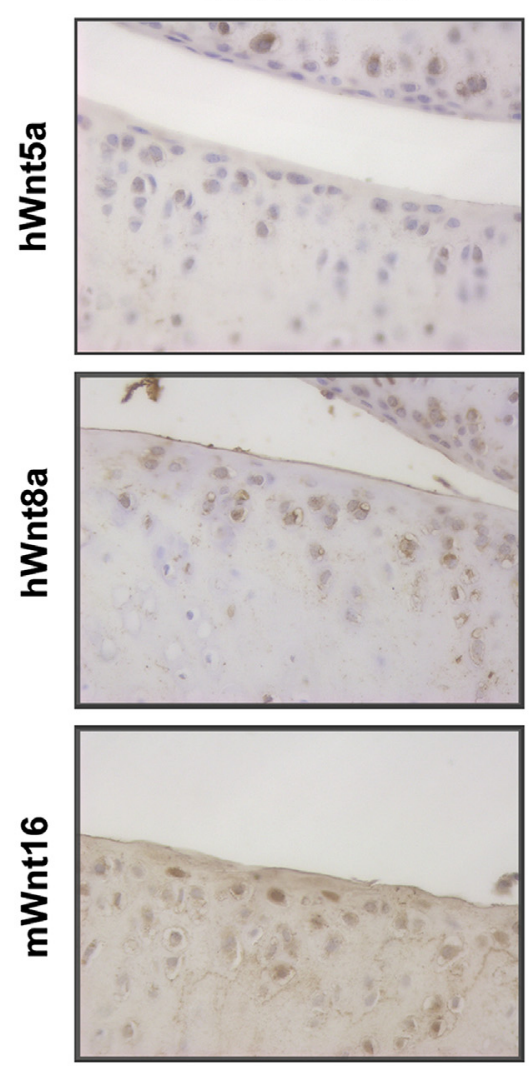

B

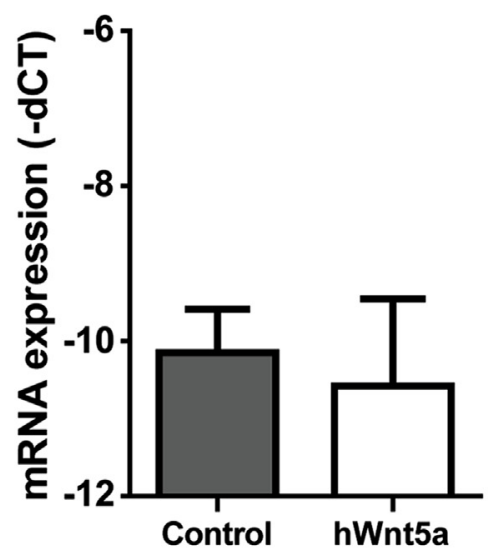

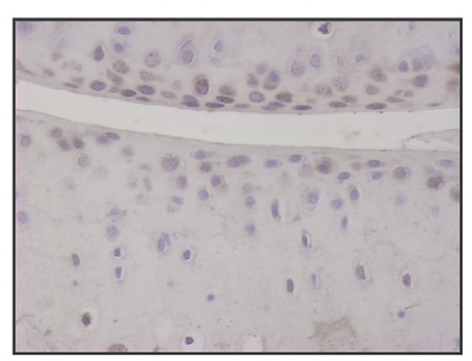
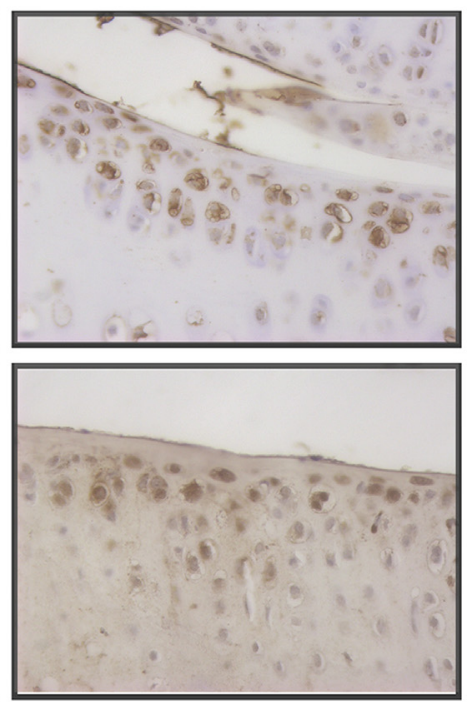

mAxin2

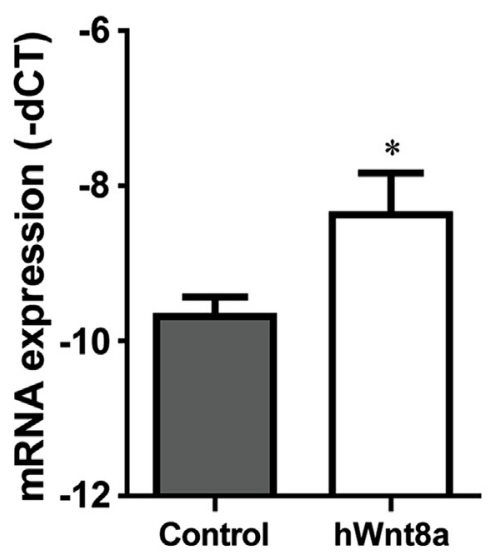

Figure 2 Synovial overexpression of Wnt8a and Wnt16, but not Wnt5a, resulted in the accumulation of $\beta$-catenin. A: Immunohistochemical staining for $\beta$-catenin showed that adenoviral overexpression of Wnt8a and Wnt16 results in increased staining in the cartilage 7 days after overexpression. By contrast, overexpression of Wnt5a had no effect. B: Quantitative real-time PCR showed that accumulation of $\beta$-catenin after overexpression of Wnt8a and Wnt16 results in increased expression of Axin2 in the cartilage. By contrast, overexpression of Wnt5a had no effect. Representative images of $\beta$-catenin staining are shown. Values are presented as $-\mathrm{dC}_{\mathrm{T}}$ means $\pm \mathrm{SEM}$. ${ }^{*} P<0.05$ versus control.

\section{Construction of Adenoviral Vectors}

To overexpress members of the Wnt signaling pathway, we generated adenoviral vectors for selected members of the Wnt signaling pathway, using a method described previously. ${ }^{25}$ We chose Wnt5a as a classical example of a Wnt ligand that signals via noncanonical signaling, Wnt16 and WISP1 as found overexpressed during experimental OA, and Wnt8a as a classical example of a Wnt ligand that signals via the canonical pathway. ${ }^{26,27}$
The complete coding sequences of Wnt5a and Wnt8a were cloned from human cDNA, whereas the complete coding sequences of Wnt16 and WISP1 were cloned from murine cDNA. The Dickkopf-related protein 1 (DKK-1) adenovirus was a kind gift of Jill Helms (Stanford University, Stanford, CA).

To achieve overexpression in vivo, we intra-articularly injected $1 \times 10^{7}$ plaque-forming units of the adenoviral vectors in a total volume of $6 \mu \mathrm{L}$ into murine knee joints. An adenoviral vector containing luciferase was used as 
A
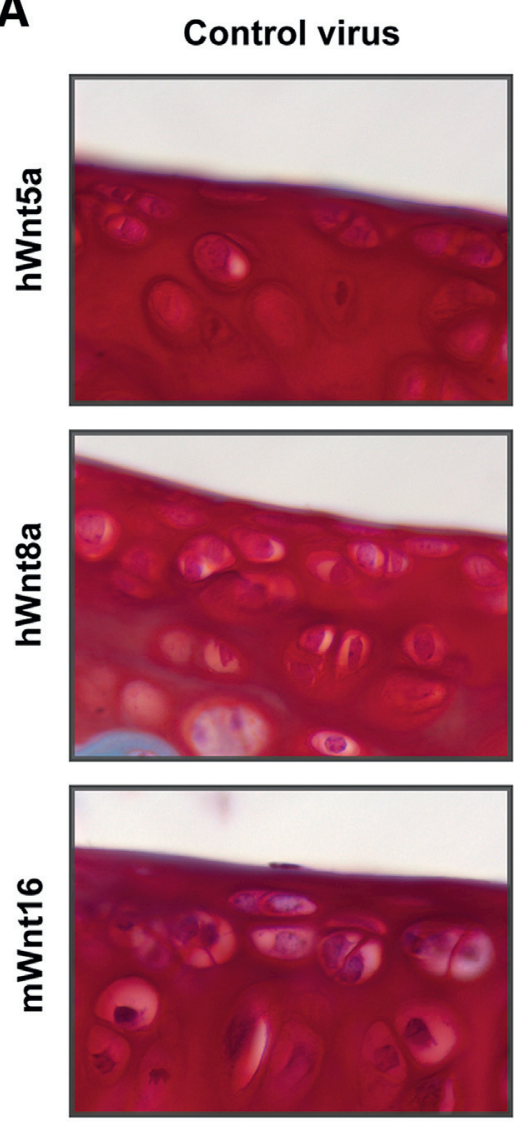

B
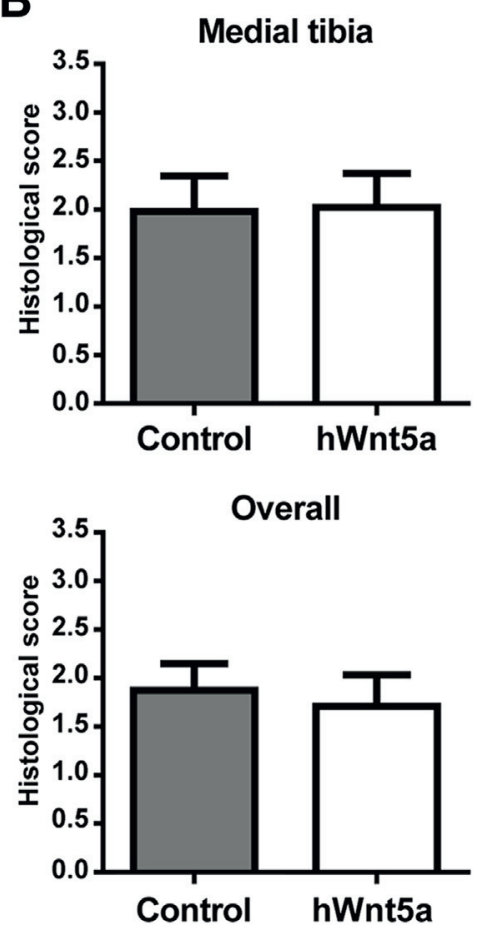

Wnt virus
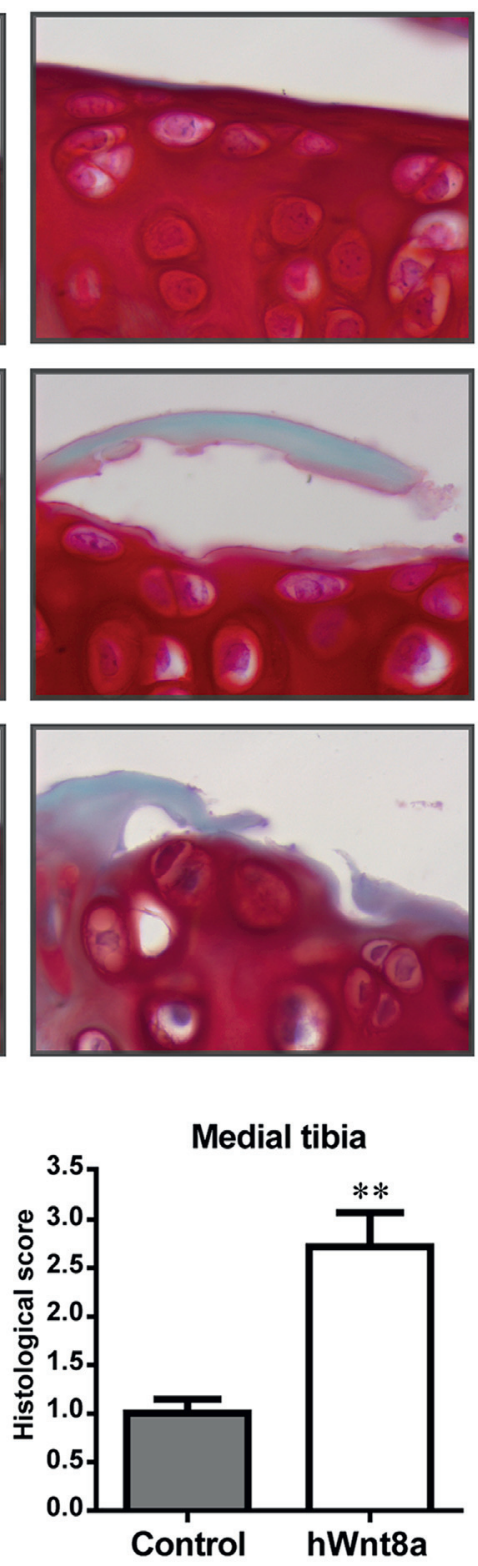

Overall

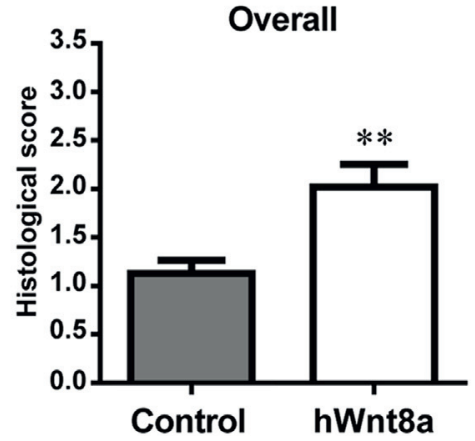

Figure 3 Synovial overexpression of Wnts that results in canonical signaling increased cartilage damage. A: Histological staining with Safranin 0 and Fast Green showed that synovial overexpression of Wnt8a and Wnt16, but not Wnt5a, results in superficial erosive cartilage lesions, mainly found at the medial tibia near the cruciate ligaments at day 7 after overexpression. B: Scoring of the cartilage damage using the Pritzker scoring system, modified for mouse, showed that canonical Wnt signaling induced by Wnt8a and Wnt16 significantly increases cartilage damage at the medial tibia and at all four cartilage areas in the tibiofemoral joint, whereas noncanonical Wnt signaling by Wnt5a overexpression did not increase the cartilage damage. Representative histological images are shown. Cartilage damage is presented as mean histological osteoarthritis scores \pm SEM. ${ }^{*} P<0.05,{ }^{* *} P<0.01$ versus control.
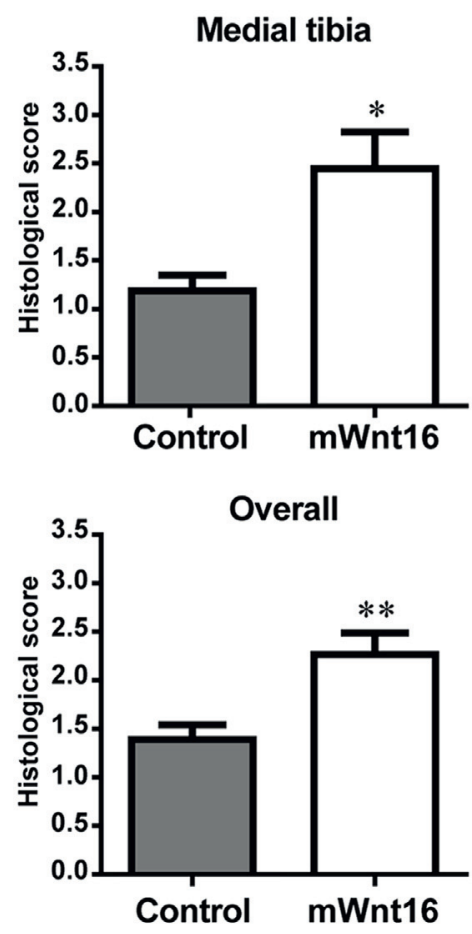

control. To determine which cells are targeted by our adenoviral vectors in vivo, we used an adenoviral vector with the same backbone containing LacZ and performed an $\mathrm{X}-\mathrm{Gal}$ staining afterward. In the experiment described with the double overexpression of two targets, $1 \times 10^{7}$ plaqueforming units of each target were injected simultaneously in a total volume of $6 \mu \mathrm{L}$. To overexpress the target in vitro, we transduced primary synovial fibroblast with the 
adenoviral vectors under serum-free condition for 2 hours. We used a multiplicity of infection of 50 for the in vitro experiments.

\section{RNA Isolation and $\mathrm{qPCR}$}

Gene expression was determined using quantitative real-time PCR (qPCR). Tissue samples for RNA isolation were homogenized using the MagNA Lyser Instrument (Roche, Mannheim, Germany). Total RNA was isolated using the RNeasy Fibrous Tissue Mini Kit (Qiagen, Venlo, the Netherlands) and subsequently reverse transcribed into cDNA. qPCR reactions were performed using the Applied Biosystems StepOnePlus realtime PCR system (Applied Biosystems, Foster City, CA). Primers were accepted if the deviation of the primer's slope was $\leq 0.3$ when compared with the slope of the GAPDH mRNA as a reference. Primer sequences were as follows: murine GAPDH, forward 5'-ATCTTCTTTTGCGTCGCCAG$3^{\prime}$ and reverse $5^{\prime}$-TTCCCCATGGTGTCTGAGC-3'; human Wnt5a, forward $5^{\prime}$-AACTCGCCCACCACACAAG- $3^{\prime}$ and reverse $\quad 5^{\prime}$-CTCATTGCGCACGCAGTAGT- $3^{\prime} ;$ human Wnt8a, forward $5^{\prime}$-ATAACAGGTCCCAAGGCCTATCT-3' and reverse $5^{\prime}$-GCACTCCTCGATGCCACTCT-3'; murine Wnt16, forward 5'-CCAAGGAGACAGCATTCATTTATG- $3^{\prime}$ and reverse $5^{\prime}$-CTGCATGACCTGGTGACAGAG-T-3'; murine WISP1, forward 5'-GCCCGAGGTACGCAATAGG- $3^{\prime}$ and reverse $5^{\prime}$-GCAGTTGGGTTGGAAGGACT-3'; and murine Axin2, forward 5'-AACGACAGCGAGTTATCCA- $3^{\prime}$ and reverse $5^{\prime}$-AGGAGGGACTCCATCTACG-3' ${ }^{\prime}$. qPCR reactions were performed in a total volume of $10 \mu \mathrm{L}$, containing $3 \mu \mathrm{L}$ cDNA, $1 \mu \mathrm{L}$ forward primer, 1 $\mu \mathrm{L}$ reverse primer, and $5 \mu \mathrm{L}$ SYBR Green Master Mix (Applied Biosystems). Reactions were presented as $-\mathrm{dC}_{\mathrm{T}}$ values, calculated by correcting the negative threshold cycle $\left(-\mathrm{C}_{\mathrm{T}}\right)$ of the gene of interest to the $-\mathrm{C}_{\mathrm{T}}$ of the reference gene $G A P D H$. qPCR analyses were performed on at least six mice per group.

\section{Western Blot}

Western blot analysis was performed to determine protein production by the adenoviruses. Total cell lysates were made and subsequently sonicated. Protein concentrations in the samples were determined with the BCA protein assay kit (Thermo Scientific, Rockford, IL). Equal protein concentrations of the samples were loaded on a SDSpolyacrylamide gel, and after separation, proteins were transferred onto nitrocellulose membranes with the iBlot system (Invitrogen). Nitrocellulose membranes were incubated with primary antibodies against Wnt5a (1:400), Wnt8a (1:1000), and WISP1 (1:1000; all R\&D systems, Minneapolis, MN) and Wnt16 (1:250; Santa Cruz Biotechnology, Santa Cruz, CA) overnight at $4^{\circ}$ C. Subsequently, membranes were incubated with appropriate secondary antibodies, and proteins were visualized using the Licor Odyssey scanner (Licor Biosciences, Lincoln,
NE) or the ECL Plus Western blotting detection system (GE Healthcare, Little Chalfont, UK), respectively. Presented Western Blots are representative images for multiple experiments.

\section{Histological Analysis}

Seven days after overexpression, mice were sacrificed, and knee joints were fixed for 7 days in $4 \%$ phosphate-buffered formalin, followed by decalcification in 5\% formic acid for 7 days. Subsequently, the joints were dehydrated and embedded in paraffin. Coronal tissue sections of $7 \mu \mathrm{m}$ were made, deparaffinized, rehydrated, and stained with Safranin $\mathrm{O}$ and Fast Green (Brunschwig, Amsterdam, the Netherlands). Eight sections from various depths were used, representing the whole knee joints.

Cartilage damage in the tibia-femoral joints was scored using the scoring system developed by Pritzker et al, ${ }^{28}$ which was modified for mice. Five sections per knee joint were scored. Histological analysis was performed for at least 10 mice per group.

\section{Immunohistochemistry}

To visualize the aggrecan neoepitopes VDIPEN and NITEGE, the result of aggrecan cleavage by MMPs and ADAMTSs, respectively, immunohistochemical staining of total knee joint sections was performed using specific antibodies directed against these cleavage sites as previously described. ${ }^{29}$ Both primary antibodies were a kind gift from John Mort (McGill University, Montreal, QC, Canada).

\section{Statistical Analysis}

Statistics were performed using GraphPad Prism version 5.0 (GraphPad Software, San Diego, CA). Differences between groups were tested using an unpaired two-tailed $t$-test. $P<0.05$ was considered significant. Results are expressed as means \pm SEM.

\section{Results}

\section{Expression of Wnts in Vivo in Osteoarthritic Tissue}

We found in microarray data from a previous study that both Wnt16 and WISP1, a downstream protein of canonical Wnt signaling, were significantly up-regulated in the synovium of a collagenase-induced OA and a Destabilization of the Medial Meniscus model longitudinal expression study. ${ }^{30}$ We validated these findings using qPCR. In the collagenase-induced $\mathrm{OA}$, we found an up-regulation of Wnt16 [fold increase at day 7 (d7), 257.7×; d21, 20.8×] and WISP1 (fold increase d7, 180.8×; d21, 39.0×). In addition, we found an up-regulation of Wnt16 (fold increase d7, 1.1×; d28, 2.9×) and WISP1 (fold increase d7, 3.2×; $\mathrm{d} 28,16.3 \times)$ in the Destabilization of the Medial Meniscus 
A NITEGE
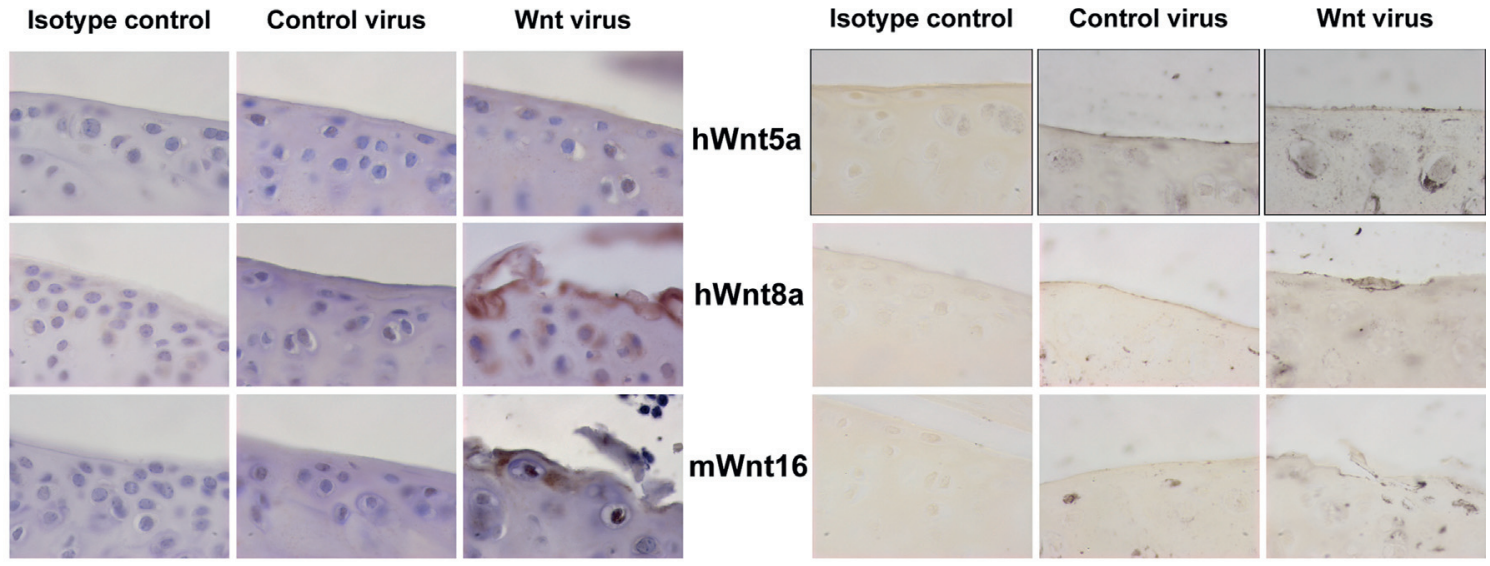

B

SafO

NITEGE

VDIPEN
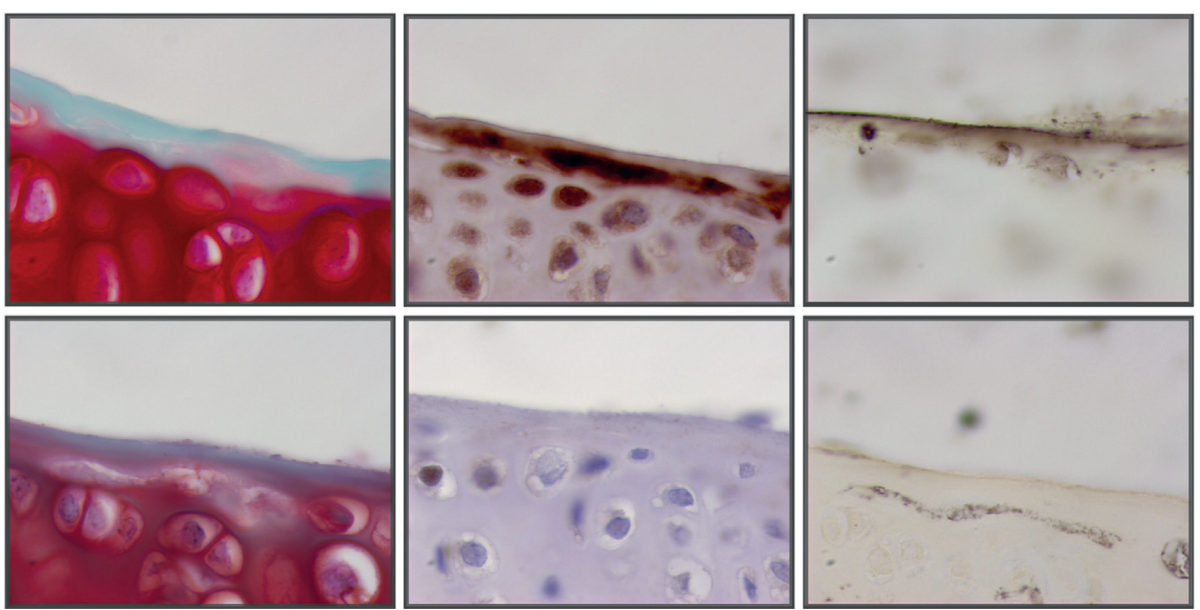

Figure 4 0steoarthritis-like cartilage pathologies resulted from protease activity. A: Immunohistochemical staining for the aggrecanase neoepitope NITEGE and the matrix metalloproteinase (MMP) neoepitope VDIPEN showed that the superficial erosive cartilage lesions induced by the overexpression of Wnt8a and Wnt16 are associated with strong NITEGE staining, whereas there was only a minor increase in VDIPEN. B: In addition to the superficial erosive cartilage lesions, several other types of pathologies in the cartilage after overexpression of Wnt8a or Wnt16, resulting in canonical signaling, were observed. Staining for NITEGE and VDIPEN showed that profound superficial proteoglycan depletion is associated with both aggrecanase and MMP activity. In addition, tears in the cartilage were associated mainly with MMP activity as measured by VDIPEN staining. Representative images of all staining are shown after overexpression of Wnt8a or Wnt16. Saf0, Safranin 0.

model. However, the effect of synovial overexpression of the members of Wnt signaling pathway on OA pathology remained unknown.

\section{Intra-Articular Injection of Adenoviral Vectors for Wnt5a, Wnt8a, and Wnt16 Leads to Protein Production by Synovial Cells}

To determine whether our Wnt adenoviral overexpression system led to increased expression levels, we transduced primary murine synovial fibroblasts with adenoviral vectors for Wnt5a, considered a noncanonical Wnt ligand; Wnt8a, historically considered to be a model for canonical signaling; and Wnt16. Adenoviral overexpression led to strongly increased expression, both on the RNA (Figure 1A) and protein (Figure 1B) levels. Next, we determined whether adenoviral overexpression in vivo led to increased expression in the synovium. RNA levels of Wnt5a, Wnt8a, and Wnt16 strongly increased in vivo as compared to levels after overexpression of the luciferase control virus in the synovium (Figure 1C). Adenoviral overexpression of LacZ showed that these adenoviral vectors caused overexpression specifically in the synovium, but not in the cartilage (Figure 1D).

\section{Overexpression of Wnt8a and Wnt16 Results in Canonical Wnt Signaling}

To check whether in our situation, synovial overexpression of the Wnt proteins led to canonical Wnt signaling in articular cartilage, we determined $\beta$-catenin accumulation as a telltale sign of canonical Wnt signaling. 

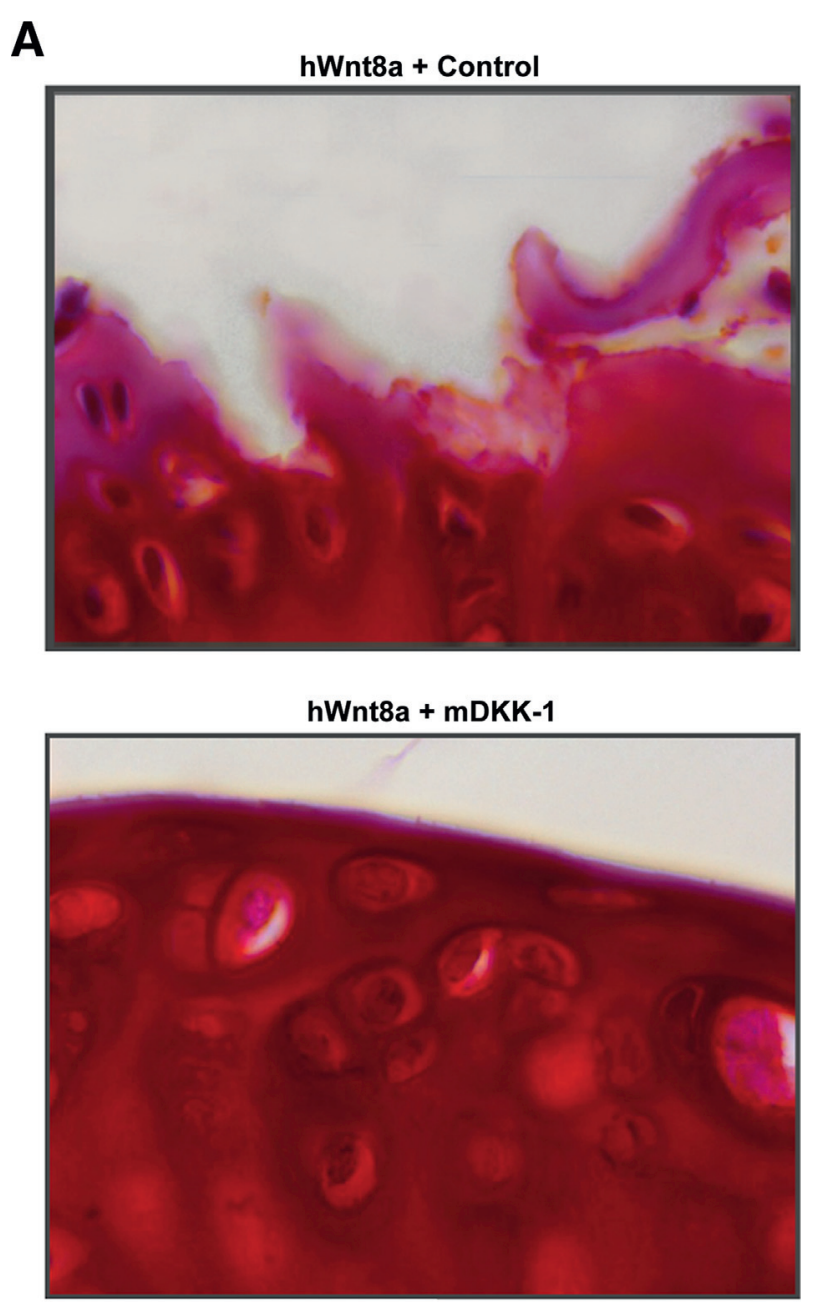

B

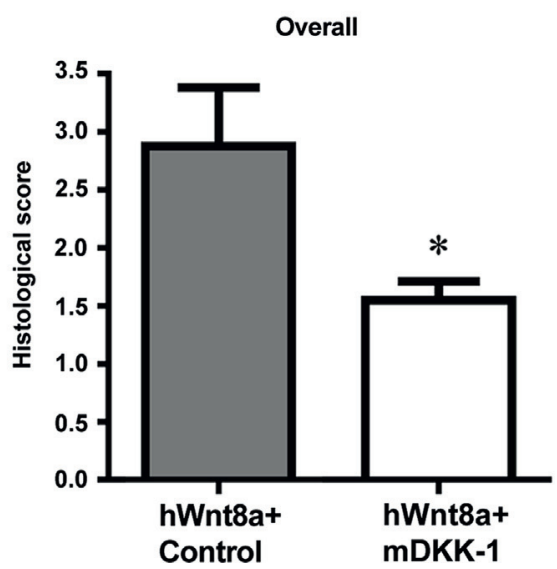

Figure 5 Inhibition of canonical Wnt signaling by Dickkopf-1 (DKK-1) overexpression reduced the cartilage damage. A: Histological staining with Safranin 0 and Fast Green showed that synovial overexpression of DKK-1 reduces the superficial erosive cartilage lesions induced by canonical Wnt signaling at day 7 after overexpression. B: Scoring of the cartilage damage using the Pritzker scoring system, modified for mouse, showed that inhibition of canonical Wnt signaling by overexpression of the selective inhibitor DKK-1 significantly reduces the cartilage damage induced by canonical Wnt signaling in the tibiofemoral joint. Representative histological images are shown. Cartilage damage is presented as mean histological osteoarthritis scores \pm SEM. ${ }^{*} P<0.05$ versus control.
Wnt8a and Wnt16, but not Wnt5a, resulted in increased levels of $\beta$-catenin in the cartilage, suggesting synovial production of both Wnt8a- and Wnt16-induced canonical Wnt signaling in the cartilage, whereas Wnt5a did not (Figure 2A). However, accumulation of $\beta$-catenin does not necessarily cause active canonical Wnt signaling. Therefore, we determined whether the signaling marker of canonical Wnt signaling Axin2 was increased. Increased expression of Wnt8a and Wnt16, but not Wnt5a, resulted in significantly increased expression of Axin2 in the cartilage (Figure 2B). These data indicate that the Wnt proteins that were produced in the synovium were able to migrate toward the cartilage to allow Wnt signaling.

\section{Canonical Wnt Signaling Causes Erosive Superficial OA-Like Cartilage Lesions}

Next, we determined whether synovial overexpression of members of the Wnt signaling pathway resulted in OA pathology. Overexpression of Wnt8a and Wnt16 led to $\beta$-catenin-dependent Wnt signaling and strongly increased the incidence of erosive lesions in the superficial cartilage layer, mainly on the medial tibia near the cruciate ligaments (control, 30\%; Wnt5a, 33\%; Wnt8a, $65 \%$; Wnt $16,64 \%$ ). Lesions were formed on the surface or just through the most superficial chondrocyte layer and often ran over a considerable length through the superficial cartilage layer (Figure 3A). We scored the cartilage damage of the tibia-femoral joints (Figure 3B). As suggested by the highly increased number of erosive lesions on the medial tibia, the most significant increase in cartilage damage was found in this part of the tibia-femoral joint. Although histological scoring of the cartilage damage in the medial and lateral femur and lateral tibia only gave a trend toward increased cartilage damage, the cumulative score of all four cartilage areas in the tibiofemoral joint was significantly increased if compared with overexpression of the control virus in the same experiments.

By contrast, noncanonical Wnt signaling, achieved by overexpression of Wnt5a, did not result in increased incidence of the OA-like cartilage lesions. Quantification of the cartilage damage using the OARSI scoring system optimized for mice did not show increased damage after overexpression of Wnt5a (Figure 3).

\section{Cartilage Damage Induced by Canonical Wnt Signaling Is Associated with Protease Activity}

We observed decreased Safranin $O$ staining around the erosive cartilage lesions, suggesting that the lesions might be the result of increased protease activity, in turn leading to degradation of the matrix surrounding the chondrocytes. Therefore, we determined the formation of the NITEGE neoepitope, which is induced by activity of the aggrecanases, using immunohistochemical staining. We observed increased NITEGE staining adjoining the erosive superficial 
cartilage lesions after overexpression of both Wnt8a and Wnt16 but not after overexpression of Wnt5a (Figure 4A). Next to the group of aggrecanases, MMP activity can result in decreased Safranin O staining by cleavage of aggrecan. We conducted immunohistochemical staining for the MMPinduced VDIPEN neoepitope. We found that canonical Wnt signaling resulted in marginally increased VDIPEN staining bordering the lesions. As positive controls, we used the growth plates, which showed strong staining (data not shown).

In addition to the superficial erosive cartilage lesions (Figure 3), we observed several other kinds of pathologies in the cartilage as the result of overexpression of both Wnt8a and Wnt16 (Figure 4B). The canonical Wnt signaling resulted in profound proteoglycan depletion in the superficial cartilage layer. This depletion was associated with both strong NITEGE and VDIPEN staining, indicating both aggrecanase and MMP activity. Tears were observed deeper in the cartilage. Staining of consecutive sections for the NITEGE and VDIPEN neoepitopes showed that this type of OA-like cartilage damage was mainly associated with MMP activity, whereas hardly any NITEGE staining was found (Figure 4).

\section{Blocking Canonical Wnt Signaling Prevents Cartilage Damage}

The data above suggest that the observed cartilage damage was the effect of Wnt signaling via the canonical $\beta$-catenindependent route. Therefore, to ensure that specifically signaling via the canonical pathway resulted in cartilage damage, we overexpressed DKK-1 to block canonical Wnt signaling. DKK-1 overexpression led to decreased severity of the lesions in the tibiofemoral joint (Figure 5A). Histological scoring showed significantly decreased cartilage damage (Figure 5B). These observations suggested that the effects on the cartilage were indeed the result of canonical Wnt signaling.

\section{WISP1 Can Be Responsible for the Effects of Canonical} Wnt Signaling

We overexpressed WISP1 to determine whether its overexpression caused cartilage lesions resulting from increased canonical Wnt signaling. Overexpression of WISP1 increased production of WISP1 at RNA level in vitro in primary murine synovial fibroblasts (Figure 6A), at protein level (Figure 6C), and in vivo in the synovium (Figure 6B). We observed an increased incidence of the same type of erosive superficial cartilage lesions that we observed after overexpression of Wnt8a and Wnt16 (53\% after WISP1 overexpression compared to $30 \%$ after overexpression of the control virus) (Figure 6D). Histological scoring showed that the cartilage damage significantly increased both the medial tibia and the overall joint score (Figure 6E). These lesions were mainly associated with increased aggrecanase activity, determined by NITEGE
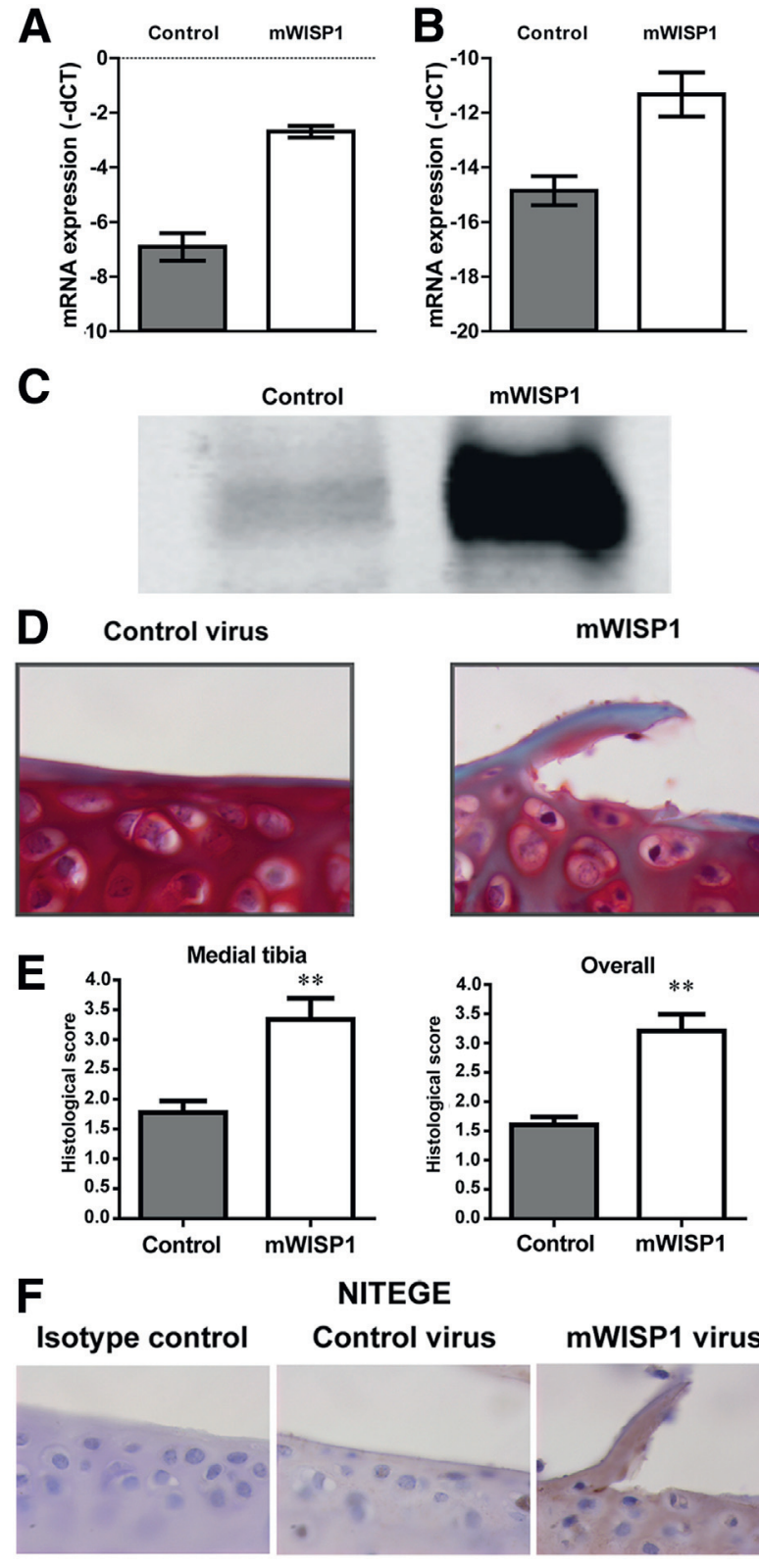

NITEGE
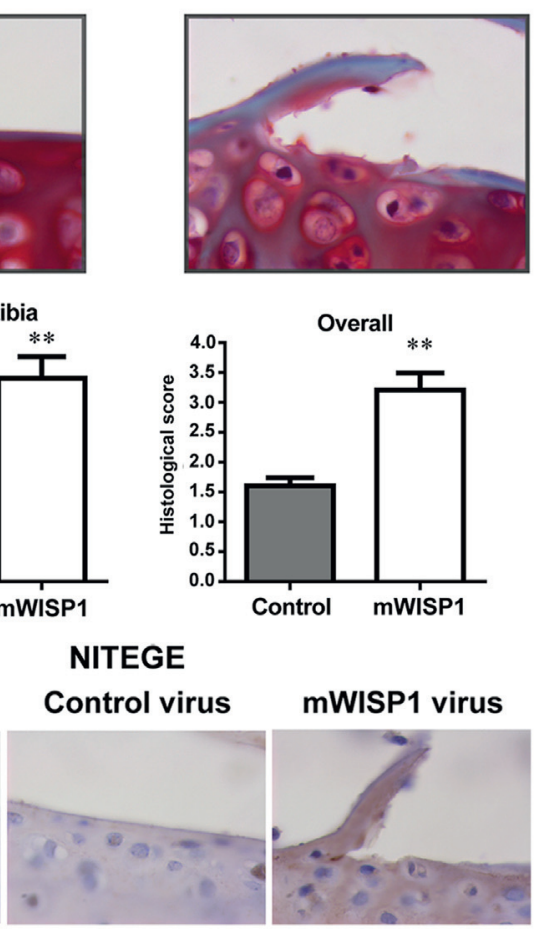

\section{VDIPEN}

Isotype control Control virus

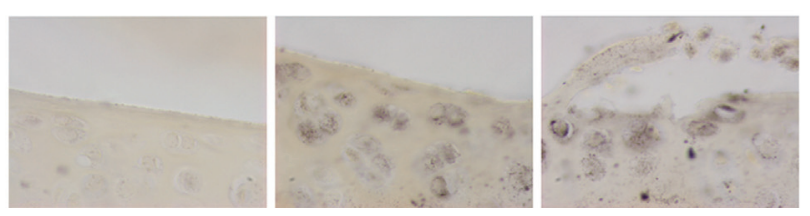

Figure 6 WISP1, a downstream protein of canonical Wnt signaling, might be partially responsible for the osteoarthritis-like cartilage pathology. A-C: WISP1 was successfully overexpressed with an adenoviral vector both in vitro in murine synovial fibroblasts ( $\mathbf{A}$ and $\mathbf{C}$ ) and in vivo in synovial tissue (B). D and $\mathbf{E}$ : An increased incidence of superficial erosive cartilage lesions at the medial tibia after overexpression of WISP1 (D) leads to significantly increased cartilage damage (E). F: The lesions are associated with increased NITEGE staining, whereas VDIPEN staining is only increased to a minor extent. Representative Western blots and images are shown. Gene expression is presented as $-\mathrm{dC}_{\mathrm{T}}$ means \pm SEM. Cartilage damage is presented as mean histological osteoarthritis scores \pm SEM. ${ }^{* *} P<0.01$ versus control. 
staining (Figure 6F). However, VDIPEN staining did not show an increase.

\section{Discussion}

Here, we show for the first time that synovial overexpression of Wnt proteins leading to canonical signaling results in increased protease activity and OA-like cartilage damage.

Wnt signaling has been linked to OA incidence via the $\beta$-catenin-dependent canonical signaling pathway. We have earlier shown that the expression of various members of the Wnt signaling pathway increases in two experimental OA mouse models. ${ }^{7}$ Therefore, in this study, we determined the effects of overexpression of selected members of the Wnt signaling pathway in vivo.

We used Wnt8a as a classical example of a Wnt ligand that signals via the canonical $\beta$-catenin-dependent pathway. ${ }^{27}$ Overexpression of Wnt8a and Wnt16 led to increased $\beta$-catenin protein levels in the articular cartilage thereby indicating signaling via the canonical pathway. Overexpression of Wnt5a, on the contrary, did not result in $\beta$-catenin accumulation. Historically, Wnt signaling pathways were divided in the $\beta$-catenin-dependent canonical Wnt signaling pathway and various $\beta$-catenin-independent noncanonical pathways. Wnt5a was considered to be a noncanonical Wnt protein that solely signaled via noncanonical Wnt signaling.

However, currently, the activation of the signaling pathways is considered to be mainly dependent on the cellular context and the arrangement of the Wnt receptors, rather than on a sequence or structural basis in the Wnt proteins. The idea of canonical and noncanonical Wnt proteins is waning, although most proteins preferentially activate either the $\beta$-catenin-dependent or -independent pathway. ${ }^{31}$ Our study shows that Wnt5a in the joint microenvironment does not result in canonical Wnt signaling.

Increased expression in the synovium of Wnt8a and Wnt16, both signaling via the canonical pathway, led to superficial erosive cartilage lesions and increased cartilage damage, as soon as 7 days after overexpression. This was observed both in young mice ( 12 weeks old) and older mice ( 6 months old, data not shown). This is consistent with findings by $\mathrm{Zhu}$ et $\mathrm{al}^{13}$ showing that the activation of $\beta$-catenin in articular chondrocytes results in increased cartilage erosion. However, we did not find significant cartilage loss in our model. This could be due to the relatively short time of overexpression of the Wnts. We observed cartilage lesions 7 days after overexpression of the adenoviral vectors, whereas $\mathrm{Zhu}$ et $\mathrm{al}^{13}$ reported these months after the conditional activation of $\beta$-catenin in the chondrocytes. However, whereas $\mathrm{Zhu}$ et $\mathrm{al}^{13}$ conditionally activated $\beta$-catenin directly in the chondrocytes, here we show that an increased expression of Wnt proteins in the synovium is able to induce canonical Wnt signaling and subsequent cartilage damage in the cartilage. However, it cannot be ruled out that the effects in the synovium as the result of synovial overexpression of canonical Wnts contribute to the effects seen in the cartilage. This underlines the idea that the synovium is an active player in the induction of OA pathology. Moreover, we tested whether the observed cartilage pathology was due to the induction of WISP1, a downstream protein of canonical Wnt signaling, reported to be increased in both the cartilage and the synovium. ${ }^{7}$ We observed an increased incidence of cartilage lesions that were comparable to overexpression of Wnt8a and Wnt16. In addition, this resulted in significantly higher cartilage damage, suggesting that WISP1 might be the downstream mediator of canonical Wnt signaling responsible for the cartilage damage.

Wnt signaling has been linked to the balance of bone formation and resorption and has been linked to fibrosis in kidney, liver, lungs, and skin; both phenomena are examples of typical OA pathologies. ${ }^{32-35}$ Luyten and collegues $^{12}$ showed increased bone mass on $\operatorname{Frz} B$ knockout in mice, whereas $\mathrm{Zhu}$ et $\mathrm{al}^{13}$ reported increased osteophyte formation after conditional activation of $\beta$-catenin in the cartilage. On the other hand, tumor necrosis factor- $\alpha$-induced DKK-1 expression and subsequent inhibition of Wnt signaling leads to decreased bone apposition, ${ }^{36}$ whereas increased expression of DKK-1 in the cartilage reduces the osteophyte size. ${ }^{37}$ However, we did not find osteophyte formation by overexpression of Wnts or WISP1 in the synovium.

Transforming growth factor- $\beta$ (TGF- $\beta$ ) is one of the dominant factors in synovial fibrosis. ${ }^{38,39}$ There is complex crosstalk shown between the Wnt and the TGF- $\beta$ signaling pathways. ${ }^{40} \mathrm{Wnt}$ signaling plays an important role in the pathogenesis of many fibrotic disorders, at least partially via interplay with the TGF- $\beta$ signaling pathway. ${ }^{41}$ However, overexpression of Wnt or WISP1 proteins in the synovium did not result in increased fibrosis in our experiments. The absence of both new bone formation and fibrosis could possibly be explained by the fact that $\mathrm{OA}$ is a composite disease with extra factors that are missing in this study.

Finally, IL-1 has been implicated in OA. Overexpression of IL-1 in the knee joint using adenovirus leads to increased inflammation in the synovial lining and depletion of both proteoglycans and type II collagen in the cartilage. ${ }^{42}$ In our study, adenoviral overexpression of members of the Wnt signaling pathway did not result in severe proteoglycan depletion throughout the cartilage, although we found focal depletion as the result of MMP and aggrecanase activity in the superficial cartilage layer, suggesting that canonical Wnt signaling did not conduct its effects via IL-1.

\section{Conclusion}

In conclusion, we have shown for the first time that excessive production of members of the Wnt signaling pathway in the synovium that signal via the canonical Wnt signaling pathway results in OA-like cartilage lesions shortly after overexpression. Moreover, we show that WISP1 might be a pivotal downstream mediator of canonical Wnt signaling in this process. 


\section{Acknowledgments}

We thank Jill Helms (Stanford University, Stanford, CA) for the DKK-1 adenovirus, John Mort (McGill University, Montreal, QC, Canada) for the antibodies directed against the neoepitopes VDIPEN and NITEGE, and Peter Newham and Sarah Brockbank (AstraZeneca, Macclesfield, UK) for their collaboration in generating the adenoviral vectors containing Wnt5a and Wnt8a.

M.H.vdB., A.N.B., P.L.vL., W.B.vdB., and P.M.vdK. developed the study concept and design; M.H.vdB. and A.W.S. performed all experiments; M.H.vdB. analyzed all data, generated all figures, and wrote the manuscript; M.H.vdB., A.B.B., A.SW., M.I.K., F.A.vdL., W.B.vdB., P.L.vL., and P.M.vdK. interpreted data and read and approved the final manuscript.

\section{References}

1. Yamagami T, Molotkov A, Zhou CJ: Canonical Wnt signaling activity during synovial joint development. J Mol Histol 2009, 40:311-316

2. Hartmann C, Tabin CJ: Dual roles of Wnt signaling during chondrogenesis in the chicken limb. Development 2000, 127:3141-3159

3. Guo X, Day TF, Jiang X, Garrett-Beal L, Topol L, Yang Y: Wnt/betacatenin signaling is sufficient and necessary for synovial joint formation. Genes Dev 2004, 18:2404-2417

4. Witte F, Dokas J, Neuendorf F, Mundlos S, Stricker S: Comprehensive expression analysis of all Wnt genes and their major secreted antagonists during mouse limb development and cartilage differentiation. Gene Expr Patterns 2009, 9:215-223

5. Luyten FP, Tylzanowski P, Lories RJ: Wnt signaling and osteoarthritis. Bone 2009, 44:522-527

6. Goldring MB, Marcu KB: Cartilage homeostasis in health and rheumatic diseases. Arthritis Res Ther 2009, 11:224

7. Blom AB, Brockbank SM, van Lent PL, van Beuningen HM, Geurts J, Takahashi N, van der Kraan PM, van de Loo FA, Schreurs BW, Clements K, Newham P, van den Berg WB: Involvement of the Wnt signaling pathway in experimental and human osteoarthritis: prominent role of Wnt-induced signaling protein 1. Arthritis Rheum 2009, 60: $501-512$

8. Loughlin J, Dowling B, Chapman K, Marcelline L, Mustafa Z, Southam L, Ferreira A, Ciesielski C, Carson DA, Corr M: Functional variants within the secreted frizzled-related protein 3 gene are associated with hip osteoarthritis in females. Proc Natl Acad Sci U S A 2004, 101:9757-9762

9. Valdes AM, Loughlin J, Oene MV, Chapman K, Surdulescu GL, Doherty M, Spector TD: Sex and ethnic differences in the association of ASPN, CALM1, COL2A1, COMP, and FRZB with genetic susceptibility to osteoarthritis of the knee. Arthritis Rheum 2007, 56: 137-146

10. Min JL, Meulenbelt I, Riyazi N, Kloppenburg M, HouwingDuistermaat JJ, Seymour AB, Pols HA, van Duijn CM, Slagboom PE: Association of the Frizzled-related protein gene with symptomatic osteoarthritis at multiple sites. Arthritis Rheum 2005, 52:1077-1080

11. Urano T, Shiraki M, Narusawa K, Usui T, Sasaki N, Hosoi T, Ouchi Y, Nakamura T, Inoue S: Q89R polymorphism in the LDL receptorrelated protein 5 gene is associated with spinal osteoarthritis in postmenopausal Japanese women. Spine (Phila Pa 1976) 2007, 32:25-29

12. Lories RJ, Peeters J, Bakker A, Tylzanowski P, Derese I, Schrooten J, Thomas JT, Luyten FP: Articular cartilage and biomechanical properties of the long bones in Frzb-knockout mice. Arthritis Rheum 2007, 56:4095-4103
13. Zhu M, Tang D, Wu Q, Hao S, Chen M, Xie C, Rosier RN, O'Keefe RJ, Zuscik M, Chen D: Activation of beta-catenin signaling in articular chondrocytes leads to osteoarthritis-like phenotype in adult beta-catenin conditional activation mice. J Bone Miner Res 2009, 24 : $12-21$

14. Zhu M, Chen M, Zuscik M, Wu Q, Wang YJ, Rosier RN, O'Keefe RJ, Chen D: Inhibition of beta-catenin signaling in articular chondrocytes results in articular cartilage destruction. Arthritis Rheum 2008, 58: 2053-2064

15. Lee MA, Park JH, Rhyu SY, Oh ST, Kang WK, Kim HN: Wnt3a expression is associated with MMP-9 expression in primary tumor and metastatic site in recurrent or stage IV colorectal cancer. BMC Cancer 2014, 14:125

16. Kwon M, Libutti SK: Filamin A interacting protein 1-like as a therapeutic target in cancer. Expert Opin Ther Targets 2014, 18:1435-1447

17. Zhao $\mathrm{C}, \mathrm{Bu} \mathrm{X}$, Zhang $\mathrm{N}$, Wang W: Downregulation of SFRP5 expression and its inverse correlation with those of MMP-7 and MT1MMP in gastric cancer. BMC Cancer 2009, 9:224

18. Schambony A, Kunz M, Gradl D: Cross-regulation of Wnt signaling and cell adhesion. Differentiation 2004, 72:307-318

19. Imai K, Morikawa M, D’Armiento J, Matsumoto H, Komiya K, Okada Y: Differential expression of WNTs and FRPs in the synovium of rheumatoid arthritis and osteoarthritis. Biochem Biophys Res Commun 2006, 345:1615-1620

20. Tamamura Y, Otani T, Kanatani N, Koyama E, Kitagaki J, Komori T, Yamada Y, Costantini F, Wakisaka S, Pacifici M, Iwamoto M, EnomotoIwamoto M: Developmental regulation of Wnt/beta-catenin signals is required for growth plate assembly, cartilage integrity, and endochondral ossification. J Biol Chem 2005, 280:19185-19195

21. Yuasa $T$, Otani $T$, Koike $T$, Iwamoto $M$, Enomoto-Iwamoto $M$ : Wnt/beta-catenin signaling stimulates matrix catabolic genes and activity in articular chondrocytes: its possible role in joint degeneration. Lab Invest 2008, 88:264-274

22. Lark MW, Bayne EK, Flanagan J, Harper CF, Hoerrner LA, Hutchinson NI, Singer II, Donatelli SA, Weidner JR, Williams HR, Mumford RA, Lohmander LS: Aggrecan degradation in human cartilage. Evidence for both matrix metalloproteinase and aggrecanase activity in normal, osteoarthritic, and rheumatoid joints. J Clin Invest 1997, 100:93-106

23. Singer II, Kawka DW, Bayne EK, Donatelli SA, Weidner JR, Williams HR, Ayala JM, Mumford RA, Lark MW, Glant TT: VDIPEN, a metalloproteinase-generated neoepitope, is induced and immunolocalized in articular cartilage during inflammatory arthritis. J Clin Invest 1995, 95:2178-2186

24. Singer II, Scott S, Kawka DW, Bayne EK, Weidner JR, Williams HR, Mumford RA, Lark MW, McDonnell J, Christen AJ, Moore VL, Mudgett JS, Visco DM: Aggrecanase and metalloproteinase-specific aggrecan neo-epitopes are induced in the articular cartilage of mice with collagen II-induced arthritis. Osteoarthritis Cartilage 1997, 5: 407-418

25. Smeets RL, Veenbergen S, Arntz OJ, Bennink MB, Joosten LA, van den Berg WB, van de Loo FA: A novel role for suppressor of cytokine signaling 3 in cartilage destruction via induction of chondrocyte desensitization toward insulin-like growth factor. Arthritis Rheum 2006, 54:1518-1528

26. Shimizu H, Julius MA, Giarre M, Zheng Z, Brown AM, Kitajewski J: Transformation by Wnt family proteins correlates with regulation of beta-catenin. Cell Growth Differ 1997, 8:1349-1358

27. Darken RS, Wilson PA: Axis induction by wnt signaling: target promoter responsiveness regulates competence. Dev Biol 2001, 234: $42-54$

28. Pritzker KP, Gay S, Jimenez SA, Ostergaard K, Pelletier JP, Revell PA, Salter D, van den Berg WB: Osteoarthritis cartilage histopathology: grading and staging. Osteoarthritis Cartilage 2006, 14: 13-29

29. Blaney Davidson EN, Vitters EL, van Lent PL, van de Loo FA, van den Berg WB, van der Kraan PM: Elevated extracellular matrix pro- 
duction and degradation upon bone morphogenetic protein-2 (BMP-2) stimulation point toward a role for BMP-2 in cartilage repair and remodeling. Arthritis Res Ther 2007, 9:R102

30. Remst DF, Blom AB, Vitters EL, Bank RA, van den Berg WB, Blaney Davidson EN, van der Kraan PM: Gene expression analysis of murine and human osteoarthritis synovium reveals elevation of transforming growth factor beta-responsive genes in osteoarthritis-related fibrosis. Arthritis Rheumatol 2014, 66:647-656

31. Willert K, Nusse R: Wnt proteins. Cold Spring Harb Perspect Biol 2012, 4:a007864

32. MacDonald BT, Joiner DM, Oyserman SM, Sharma P, Goldstein SA, He X, Hauschka PV: Bone mass is inversely proportional to Dkk1 levels in mice. Bone 2007, 41:331-339

33. Jian YC, Wang JJ, Dong S, Hu JW, Hu LJ, Yang GM, Zheng YX, Xiong WJ: Wnt-induced secreted protein 1/CCN4 in liver fibrosis both in vitro and in vivo. Clin Lab 2014, 60:29-35

34. Lam AP, Herazo-Maya JD, Sennello JA, Flozak AS, Russell S, Mutlu GM, Budinger GR, DasGupta R, Varga J, Kaminski N, Gottardi CJ: Wnt coreceptor Lrp5 is a driver of idiopathic pulmonary fibrosis. Am J Respir Crit Care Med 2014, 190:185-195

35. Beyer C, Reichert H, Akan H, Mallano T, Schramm A, Dees C, PalumboZerr K, Lin NY, Distler A, Gelse K, Varga J, Distler O, Schett G, Distler JH: Blockade of canonical Wnt signalling ameliorates experimental dermal fibrosis. Ann Rheum Dis 2013, 72:1255-1258
36. Diarra D, Stolina M, Polzer K, Zwerina J, Ominsky MS, Dwyer D, Korb A, Smolen J, Hoffmann M, Scheinecker C, van der Heide D, Landewe R, Lacey D, Richards WG, Schett G: Dickkopf-1 is a master regulator of joint remodeling. Nat Med 2007, 13:156-163

37. Oh $\mathrm{H}$, Chun $\mathrm{CH}$, Chun JS: Dkk-1 expression in chondrocytes inhibits experimental osteoarthritic cartilage destruction in mice. Arthritis Rheum 2012, 64:2568-2578

38. Bakker AC, van de Loo FA, van Beuningen HM, Sime P, van Lent PL, van der Kraan PM, Richards CD, van den Berg WB: Overexpression of active TGF-beta-1 in the murine knee joint: evidence for synoviallayer-dependent chondro-osteophyte formation. Osteoarthritis Cartilage 2001, 9:128-136

39. van Beuningen HM, van der Kraan PM, Arntz OJ, van den Berg WB: Transforming growth factor-beta 1 stimulates articular chondrocyte proteoglycan synthesis and induces osteophyte formation in the murine knee joint. Lab Invest 1994, 71:279-290

40. Guo X, Wang XF: Signaling cross-talk between TGF-beta/BMP and other pathways. Cell Res 2009, 19:71-88

41. Guo Y, Xiao L, Sun L, Liu F: Wnt/beta-catenin signaling: a promising new target for fibrosis diseases. Physiol Res 2012, 61:337-346

42. Rowan AD, Hui W, Cawston TE, Richards CD: Adenoviral gene transfer of interleukin-1 in combination with oncostatin $\mathrm{M}$ induces significant joint damage in a murine model. Am J Pathol 2003, 162: 1975-1984 